\title{
Wetting-induced interaction between rigid nanoparticle and plate: A Monte Carlo study
}

\section{AUTHOR(S):}

Shinto, H; Uranishi, K; Miyahara, M; Higashitani, K

\section{CITATION:}

Shinto, $\mathrm{H}$... [et al]. Wetting-induced interaction between rigid nanoparticle and plate: A Monte Carlo study. JOURNAL OF CHEMICAL PHYSICS 2002, 116(21): 9500-9509

\section{ISSUE DATE:}

2002-06-01

URL:

http://hdl.handle.net/2433/39737

\section{RIGHT:}

Copyright 2002 American Institute of Physics. This article may be downloaded for personal use only. Any other use requires prior permission of the author and the American Institute of Physics. 


\title{
Wetting-induced interaction between rigid nanoparticle and plate: A Monte Carlo study
}

\author{
Hiroyuki Shinto, Katsushige Uranishi, Minoru Miyahara, and Ko Higashitania) \\ Department of Chemical Engineering, Kyoto University, Yoshida, Sakyo-ku, Kyoto 606-8501, Japan
}

(Received 21 November 2001; accepted 7 March 2002)

\begin{abstract}
The interaction forces between a nanosphere and a flat plate in undersaturated vapors are examined. We perform grand canonical Monte Carlo simulations, where the surfaces of the sphere and the plate are treated as rigid smooth and the vapors are modeled as a Lennard-Jones fluid of nonpolar spherical molecules. The following results are obtained: (i) The force between the sphere and plate becomes attractive at the surface distances where capillary condensation takes place in the gap between the surfaces; (ii) the onset of the attractive force becomes farther as the relative vapor pressure increases; (iii) the curve of the pull-off force (or the adhesion force) as a function of the relative vapor pressure has a peak, where the peak position shifts to a higher relative pressure and the peak height becomes smaller with decreasing the attractive interaction of the surfaces with a fluid molecule; (iv) at the relative vapor pressure where the pull-off force becomes maximum, the coverage of the surface by fluid molecules is about 0.45 regardless of the strength of the surfacefluid attraction; in addition, the gap between the sphere and plate exhibits the same feature of wetting, that is, a circular string of fluid molecules plus an adsorbed layer on each surface. The results of our simulations will be compared in details with those of force measurements by the atomic force microscope and the surface force apparatus. (c) 2002 American Institute of Physics.
\end{abstract} [DOI: $10.1063 / 1.1473817$ ]

\section{INTRODUCTION}

While the wettability of solid surfaces and pores (adsorption and capillary condensation) is relatively well understood, the interaction between wetted bodies is still questioned. Detailed understanding and exact description of the interaction between solid surfaces both in vacuum (or dry air) and in a capillary condensed fluid have been a central subject not only in colloid and interface science, but also particle technology and tribology. From an industrial point of view, deep knowledge of surface adhesion is important in the process of particulate products such as ceramics, catalysts, and pharmaceuticals, in the manipulation of microdevices and micromachines, and in many operations (e.g., particle filter, fluidized bed, and painting and particulate coating).

The atomic force microscope (AFM) (Refs. 1 and 2) and the surface force apparatus (SFA) (Ref. 3) are the powerful experimental devices to measure the interaction forces between two surfaces in a vapor as well as in a liquid; however, our discussion here will be limited to the experiments in capillary condensed fluids. The force that is necessary to separate one surface from the other surface after establishing contact is referred to as the pull-off force (or the adhesion force). Several studies have reported the pull-off force as a function of the relative vapor pressure. AFM measurements have been performed only in a humid environment (or a water vapor); ${ }^{4,5}$ the pull-off force between a hydrophilic $\mathrm{Si}_{3} \mathrm{~N}_{4}$ tip of $10-20 \mathrm{~nm}$ radius and a flat surface of mica or silicon oxide was measured. The relationship between the

\footnotetext{
a) Author to whom correspondence should be addressed. Electronic mail: higa@cheme.kyoto-u.ac.jp
}

pull-off force and the relative humidity $(\mathrm{RH})$ was found to show three regions: (i) the force remained constant in the low-RH range, (ii) it increased with $\mathrm{RH}$ in the mid-RH range, and (iii) it decreased with $\mathrm{RH}$ in the high-RH range. In other words, the pull-off force had a peak in the mid-RH range. (This behavior can be altered by the presence of surface roughness, as observed in the pull-off forces between a silica sphere of $3.7 \mu \mathrm{m}$ radius and a flat surface of silicon oxide $^{5}$ and between two silica spheres of $0.9 \mu \mathrm{m}$ radius each. ${ }^{6}$ Note that the silica spheres had the surface roughness of about $10 \mathrm{~nm},{ }^{5}$ which was comparable to the radius of the AFM tip.) In the SFA, on the other hand, two molecularly smooth mica surfaces of about $1 \mathrm{~cm}$ radius each are employed in a cross-cylinder configuration; the radius of curvature of the surfaces is extremely larger than that of an AFM tip. Chistenson ${ }^{7}$ measured the pull-off force between the mica surfaces in a vapor of water, cyclohexane, or $n$-hexane. He reported that the pull-off force increased or decreased monotonically with the relative vapor pressure, depending on the vapor employed and the pretreatment of the mica surface; accordingly, the pull-off force had no peak. (Also Christenson ${ }^{7}$ demonstrated that the results of an earlier work by Fisher and Israelachvili ${ }^{8}$ were in error due to a technical artifact.) Thus, the results of AFM and SFA measurements seem to have nothing in common as far as the dependence of the pull-off force on the relative vapor pressure is concerned. This discrepancy could be caused by the difference of the curvature of surfaces, ${ }^{5}$ the inevitable surface deformations, ${ }^{9}$ and the undesirable surface contaminations; ${ }^{10,11}$ nevertheless, the details remain elusive.

Theoretical treatments of surface adhesion have been fo- 
cused on the deformation of surfaces in the absence and the presence of a capillary-condensable fluid; ${ }^{12-14}$ capillary condensation has been treated with the Laplace-Kelvin equation, ${ }^{15}$ where the fluid in the confined space is assumed to have the same properties as in the bulk phase. ${ }^{13}$ Alternatively, a density functional theory (DFT) treats the fluid between surfaces with a modern molecular theory. ${ }^{16,17}$ Frink and van $\mathrm{Swol}^{16}$ employed the DFT to analyze the force between rigid nondeformable surfaces in a vapor of simple molecules. Their DFT successfully captured not only the oscillatory behavior of forces observed in SFA measurements, ${ }^{18,19}$ but also the aforementioned variations of the pull-off force as a function of the relative vapor pressure, ${ }^{4,5,7}$ while the conventional theories based on the Laplace-Kelvin equation have predicted neither of them. ${ }^{13}$ This demonstrates the importance of considering the molecular nature of a fluid in the confined geometries for exact description of interaction forces between surfaces with the liquid bridge. Within the framework of DFT, however, the description of a fluid employed is more or less approximate; ${ }^{16,17}$ in fact, a simple version of DFT, where the wetting films on the surfaces were treated in a coarse-grained manner, captured neither the oscillatory behavior of the force nor the variations of the pull-off force. ${ }^{17}$ In addition, because Frink and van $\mathrm{Swol}^{16}$ analyzed the surface free energy of parallel plates in a capillary condensed fluid with no liquidvapor meniscus (or under no tensile condition) to transform it into the sphere-plate interaction force using the Derjaguin approximation, ${ }^{15}$ a liquid-vapor meniscus between the sphere and plate was treated implicitly and approximately. For these reasons, it is necessary to compare carefully the results from the DFT with those from computer simulations ${ }^{20}$ based on the molecular dynamics (MD) or the Monte Carlo (MC) method.

Only a few MD and MC studies have reported the results of interaction force between solid surfaces in the presence of a capillary condensed fluid of water ${ }^{21}$ or $n$-hexadecane, ${ }^{22}$ where no control of the relative vapor pressure was attempted. Such simulations are unfavorable for computation of the pull-off force as a function of the relative vapor pressure. Recently, we developed the canonical ensemble MD method to compute the interaction between rigid spheres immersed in various fluids such as pure simple fluid, multicomponent fluid, and complex fluid. ${ }^{23,24}$ When one employs our method to compute the interaction between the spheres in a capillary-condensable vapor, a barostat of the simulation box ${ }^{20}$ would be required to control the vapor pressure around a given value; otherwise, the vapor pressure might suffer undesirable drastic change at around the surface separation of capillary condensation. Alternatively, one can apply the grand canonical MC (GCMC) method ${ }^{20}$ straightforward to the system.

In this paper, we report a GCMC study of interaction forces between a sphere and a flat plate in undersaturated vapors; the surfaces of the sphere and the plate are smooth and rigid. The sphere-plate geometry was chosen instead of a plate-plate geometry (i.e., a slit geometry) since the former is more realistic and faithful to reproduction of a liquid bridge in the gap between the surfaces. A Lennard-Jones (LJ)
TABLE I. Systems of GCMC simulations (see also Fig. 1). ${ }^{\mathrm{a}}$

\begin{tabular}{|c|c|c|c|c|}
\hline System & $\begin{array}{c}\text { Box } \\
\text { dimension } \\
L_{x}, L_{y}, L_{z}(\sigma)\end{array}$ & $\begin{array}{c}\text { Directions } \\
\text { of periodic } \\
\text { boundary }\end{array}$ & $\begin{array}{c}\text { Radius } \\
\text { of sphere } \\
R(\sigma)\end{array}$ & $\begin{array}{c}\text { Number } \\
\text { of fluid } \\
\text { molecules } N\end{array}$ \\
\hline A & $15,15,15(20,20,20)^{\mathrm{b}}$ & $x, y, z$ & & $10-100$ \\
\hline B & $10,10,20(15,15,20)^{\mathrm{b}}$ & $x, y^{\mathrm{c}}$ & & $15-350$ \\
\hline $\mathrm{C}$ & $27,27,35$ & $x, y^{\mathrm{c}}$ & $2.5^{\mathrm{d}}$ & $130-3000$ \\
\hline
\end{tabular}

aTemperature was set to $T^{*}=k_{B} T / \varepsilon=1.0$ throughout the present simulations.

${ }^{b}$ Values in parentheses are the dimension of a larger box employed for $\mu / k_{B} T<-13.10$.

${ }^{c}$ The interactive and noninteractive walls were located at $z=0$ and $z=L_{z}$, respectively.

${ }^{\mathrm{d}}$ The sphere was immobile.

fluid of nonpolar spherical molecules was employed as a capillary-condensable vapor. We explore (A) the bulk vapors in Sec. IV A, (B) the adsorption isotherms of fluid molecules onto the isolated plate in Sec. IV B, and (C) the sphere-plate interaction forces in Sec. IV C.

\section{MODEL AND SIMULATION}

\section{A. Systems}

Table I lists three different systems employed: (A) a bulk vapor; (B) the vapor confined between two flat walls for computation of the adsorption isotherms; and (C) the confined vapor in the presence of a large immobile sphere for computation of the sphere-plate interactions. Figure 1 illustrates the simulation box of system $\mathrm{C}$, where fluid molecules and the immobile sphere were confined between two planar walls: the bottom wall interacted with fluid molecules in the same way as the sphere, while the top wall had no interaction except the excluded volume effect (i.e., the hard wall). The origin of coordinates was taken at the center of the bottom wall. The top and bottom walls had the width of $L_{x}=L_{y}$ $=27 \sigma$ each and were separated in the $z$ direction by $L_{z}$ $=35 \sigma$, where $\sigma$ denotes the diameter of a fluid molecule as will be in Eq. (1). The periodic boundary conditions were

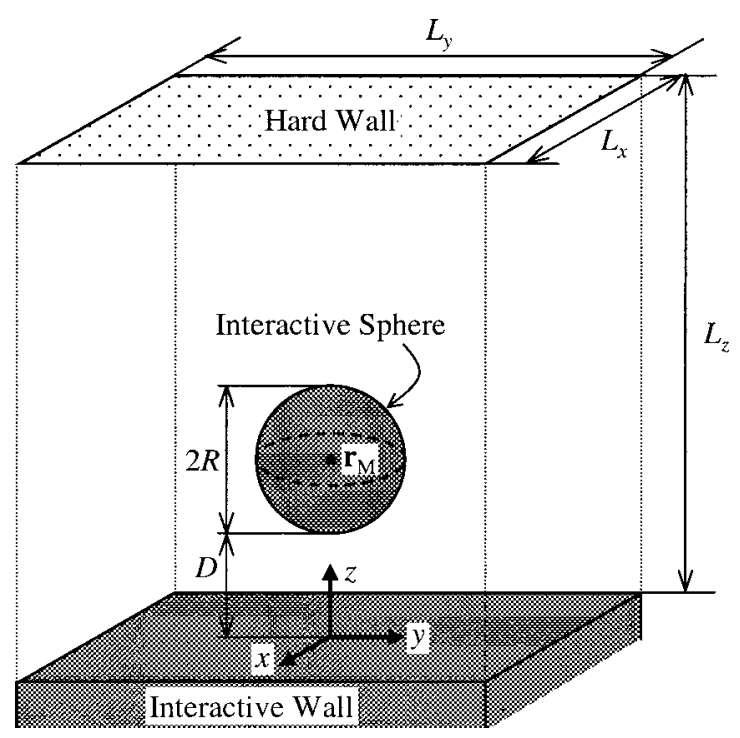

FIG. 1. A schematic of the simulation box for system C. Fluid molecules are not shown for clarity. 
applied to the $x$ and $y$ directions parallel to the walls. The radius of the sphere was set to $R=2.5 \sigma$, which seems relatively small but is large enough to capture the oscillatory behavior of sphere-plate interactions in a Lennard-Jones (LJ) fluid. ${ }^{25}$ The center of this sphere was fixed at $\mathbf{r}_{\mathrm{M}}$ $=(0,0, D+R)$ near the interactive wall at $z=0$, where $D$ is the nearest separation between the surfaces of the sphere and the interactive wall. Judging from the curvature and the geometry of the surfaces, one can consider system $\mathrm{C}$ as a model of tip-substrate force measurements by AFM. ${ }^{4,5}$

The simulation box of system B was similar to that of system $\mathrm{C}$; the differences were the absence of the sphere and the box dimension as given in Table I.

\section{B. Potential models}

For the fluid-fluid interaction, the LJ 12-6 potential,

$$
\phi_{\mathrm{FF}}\left(r_{i j}\right)=4 \varepsilon\left[\left(\frac{\sigma}{r_{i j}}\right)^{12}-\left(\frac{\sigma}{r_{i j}}\right)^{6}\right],
$$

was used, where $r_{i j}$ is the separation of two fluid molecules $i$ and $j, \varepsilon$ the fluid-fluid interaction energy parameter, and $\sigma$ is the diameter of a fluid molecule. Parameters for argon were used: $\varepsilon / k_{B}=119.8 \mathrm{~K}, \quad \sigma=0.3405 \mathrm{~nm}, \quad$ and $m$ $=39.948 \mathrm{~g} / \mathrm{mol}$, where $m$ is the mass of a fluid molecule and $k_{B}$ is the Boltzmann constant. The cutoff distance was set to $r_{\mathrm{FF}}^{\mathrm{cut}}=5 \sigma$, which seemed large enough to represent molecules with the full LJ potential; accordingly, no long-range correction was attempted.

For the planar wall interacting with a fluid molecule, the 10-4-3 potential of Steele,

$$
\begin{aligned}
& \phi_{\mathrm{WF}}\left(z_{\mathrm{W} i}\right) \\
& =2 \pi \varepsilon_{\mathrm{WF}}\left[\frac{2}{5}\left(\frac{\sigma}{z_{\mathrm{W} i}}\right)^{10}-\left(\frac{\sigma}{z_{\mathrm{W} i}}\right)^{4}-\frac{\sigma^{4}}{3 \Delta\left(z_{\mathrm{W} i}+0.61 \Delta\right)^{3}}\right]
\end{aligned}
$$

was employed, where $z_{\mathrm{W} i}$ is the perpendicular distance from the wall to molecule $i, \varepsilon_{\mathrm{WF}}$ the wall-fluid interaction energy parameter, and $\Delta=\sigma / \sqrt{2} .{ }^{26}$ On the other hand, the noninteractive wall was treated as the hard wall,

$$
\phi_{\mathrm{WF}}^{\mathrm{HW}}\left(z_{\mathrm{W} i}\right)=\left\{\begin{array}{cc}
\infty, & z_{\mathrm{W} i}<0, \\
0, & z_{\mathrm{W} i} \geqslant 0 .
\end{array}\right.
$$

The sphere of $R=2.5 \sigma$ interacting with a fluid molecule was represented by

$$
\begin{aligned}
\phi_{\mathrm{MF}}\left(r_{\mathrm{M} i}\right)= & 2 \pi \varepsilon_{\mathrm{MF}}\left[\frac{2}{5}\left(\frac{\sigma}{r_{\mathrm{M} i}-R}\right)^{10}-\left(\frac{\sigma}{r_{\mathrm{M} i}-R}\right)^{4}\right. \\
& \left.-\frac{\sigma^{4}}{3 \Delta\left(r_{\mathrm{M} i}-R+0.61 \Delta\right)^{3}}\right]
\end{aligned}
$$

where $r_{\mathrm{M} i}$ is the center-to-center distance between the sphere and molecule $i$, at $\mathbf{r}_{\mathrm{M}}$ and $\mathbf{r}_{i}$ (i.e., $r_{\mathrm{M} i}=\left|\mathbf{r}_{\mathrm{M}}-\mathbf{r}_{i}\right|$ ), $\varepsilon_{\mathrm{MF}}$ the sphere-fluid interaction energy parameter and again $\Delta$ $=\sigma / \sqrt{2}$. The cutoff distance of $r_{\mathrm{MF}}^{\mathrm{cut}}=10 \sigma+R$ was used with no long-range correction.
The direct sphere-plate interaction could be introduced as a function of the nearest surface separation $D .{ }^{27}$ In the present study, however, it was assumed to be zero for simplicity,

$$
U_{\mathrm{MW}}^{\mathrm{dir}}(D)=0 .
$$

This indicates that the sphere-plate interaction presented below is entirely attributable to the fluid-mediated interaction as will be explained in Sec. III C. The total energy of system C was

$$
\begin{aligned}
& U_{\text {system C }}=U_{\mathrm{FF}}+U_{\mathrm{WF}}+U_{\mathrm{MF}}+U_{\mathrm{MW}}^{\mathrm{dir}}, \\
& U_{\mathrm{FF}}=\sum_{i=1}^{N-1} \sum_{j>i}^{N} \phi_{\mathrm{FF}}\left(\left|\mathbf{r}_{i}-\mathbf{r}_{j}\right|\right), \\
& U_{\mathrm{WF}}=\sum_{i=1}^{N}\left\{\phi_{\mathrm{WF}}\left(z_{i}\right)+\phi_{\mathrm{WF}}^{\mathrm{HW}}\left(L_{z}-z_{i}\right)\right\}, \\
& U_{\mathrm{MF}}=\sum_{i=1}^{N} \phi_{\mathrm{MF}}\left(\left|\mathbf{r}_{\mathrm{M}}-\mathbf{r}_{i}\right|\right),
\end{aligned}
$$

where $N$ is the number of fluid molecules in the box. The total energy was $U_{\text {system A }}=U_{\mathrm{FF}}$ for system A and $U_{\text {system B }}$ $=U_{\mathrm{FF}}+U_{\mathrm{WF}}$ for system $\mathrm{B}$.

Parameters $\varepsilon_{\mathrm{MF}}$ and $\varepsilon_{\mathrm{WF}}$ correspond to the affinity of the surfaces of the sphere and the plate for a fluid molecule, respectively. The magnitude of this affinity would influence both the adsorption isotherms and the sphere-plate interactions. For exploration of this influence the parameters were set to $\varepsilon_{\mathrm{MF}}=\varepsilon_{\mathrm{WF}}=\chi \varepsilon$ : three different values of $\chi=0.5,0.7$, and 1.0 were considered. Hereafter the parameter $\chi$ is referred to as the surface affinity.

\section{Simulation method}

The grand canonical Monte Carlo (GCMC) method ${ }^{20,28}$ was used, in which the conserved properties are the chemical potential $\mu$, the volume $V$ and the temperature $T$. This method is favorable for inhomogeneous systems such as systems $\mathrm{B}$ and $\mathrm{C}$ of the present study, because it allows us to know via $\mu$ the thermodynamic state of the bulk phase in equilibrium with an inhomogeneous fluid as well as to sample efficiently by attempts of molecule creation and destruction. The temperature was set to $T^{*}=k_{B} T / \varepsilon=1.0$ throughout the present simulations. Henceforth the asterisk denotes the properties reduced by units of the LJ fluid that is represented by Eq. (1).

\section{Bulk vapors}

When $\mu$ is given, one can calculate the pressure $p$ and the density $\rho$ of the bulk vapor under the ideal gas assumption,

$$
\begin{aligned}
& \mu / k_{B} T=3 \ln \Lambda+\ln \left(p / k_{B} T\right), \\
& \rho=p / k_{B} T,
\end{aligned}
$$

where $\Lambda$ is the de Broglie thermal wavelength of $\Lambda$ $=h /\left(2 \pi m k_{B} T\right)^{1 / 2}$ and $h$ is the Planck constant. Equations (7) and (8) provide, however, only the approximate values because of the ideal gas assumption. 
To obtain the more exact values of $p$ and $\rho$ for given $\mu$, we carried out GCMC simulations of the bulk vapor for a range of $\mu / k_{B} T$ from -14.60 to -11.60 using system A. It is worth noting that the larger value of $\mu$ generally provides the higher pressure [see Eq. (7)] up to the saturated vapor pressure $p_{\text {sat }}$. The simulations used 10-100 fluid molecules in the cubic box of the side length of $L=15 \sigma$ for $\mu / k_{B} T$ $\geqslant-13.10$ or otherwise $L=20 \sigma$. The periodic boundary conditions were applied to all the directions. The length of the runs was 300000 cycles per simulation after equilibration of 100000 cycles. One cycle denotes the total $3 N$ operations: $N$ trial displacements, $N$ trial destructions, and $N$ trial creations of a molecule, where $N$ indicates the number of molecules after all the operations at the previous cycle and may vary from cycle to cycle. ${ }^{20,28}$ Each run started from the molecular positions that were randomly chosen from the face-centeredcubic (fcc) lattice points, that is, the pseudorandom configuration.

\section{Adsorption isotherms}

We performed GCMC simulations of system B for a range of $\mu$ to obtain the adsorption isotherm of the interactive plate. A sequence of the simulations was implemented for surface affinities of $\chi\left(=\varepsilon_{\mathrm{WF}} / \varepsilon\right)=0.5,0.7$, and 1.0; consequently, three isotherms were obtained for the plate of different affinities. The simulations used 15-350 fluid molecules in the box of dimension $10 \sigma \times 10 \sigma \times 20 \sigma$ for $\mu / k_{B} T$ $\geqslant-13.10$ or otherwise $15 \sigma \times 15 \sigma \times 20 \sigma$. The length of the runs was 300000 cycles per simulation after equilibration of 100000 cycles starting from the pseudorandom configuration of molecules.

\section{Surface forces}

For a GCMC simulation of system $\mathrm{C}$ depicted in Fig. 1 and Table I, one must specify three parameters: the chemical potential $\mu$, the surface affinity $\chi$, and the nearest surface separation $D$. It is difficult to simulate for the whole range of these parameters because of the computational cost. Hence, we carried out following two types of simulations.

First, using $\chi=1.0$ only, the simulations were performed for various distances of $D$; consequently, the surface force was obtained as a function of $D$ (i.e., the force-distance profile). A sequence of these simulations was conducted for $\mu / k_{B} T=-13.00,-12.20$, and -11.75 , which are equivalent to the relative vapor pressures of $p / p_{\text {sat }}=0.234,0.544$, and 0.899 , respectively as will be shown in Sec. IV A. Thus, three force-distance profiles were obtained for the different relative pressures.

Second, using $D / \sigma \approx 2$ or 3 at which the force profiles have a global minimum (i.e., the pull-off force) as will be demonstrated in Sec. IV C 1, the simulations were executed for a range of $\mu$; consequently, the pull-off force was obtained as a function of $p / p_{\text {sat }}$. After a series of these simulations was implemented for different values of $\chi$, three profiles of the pull-off force were obtained.

The simulations used 130-3000 fluid molecules in the box. The length of the runs spanned 50000-150000 cycles per simulation after equilibration of 50000-100000 cycles, where the pseudorandom configuration of molecules was used as an initial configuration. We ensure that no liquid bridge was initially observed in the gap between the sphere and plate.

\section{DATA ANALYSIS}

\section{A. Bulk vapors}

The density and the pressure of a bulk vapor at given $\mu$ are calculated as

$$
\begin{aligned}
& \rho^{*}=\rho \sigma^{3}, \quad \rho=\langle N\rangle / V, \\
& p^{*}=p \sigma^{3} / \varepsilon, \\
& p=\frac{1}{V}\left[\langle N\rangle k_{B} T-\frac{1}{3}\left\langle\sum_{i=1}^{N-1} \sum_{j>i}^{N} r_{i j} \frac{d \phi_{\mathrm{FF}}\left(r_{i j}\right)}{d r_{i j}}\right\rangle\right],
\end{aligned}
$$

respectively, where $\langle\cdots\rangle$ denotes the ensemble average of the function enclosed. Throughout the present simulations, the ensemble average was taken using the data at every fifth cycle.

\section{B. Adsorption isotherms}

The local density of fluid molecules as a function of $z$, $\rho(z)$, is

$$
\rho^{*}(z)=\rho(z) \sigma^{3}, \quad \rho(z)=\langle N(z)\rangle / A \Delta z,
$$

where $N(z)$ is the number of molecules in a slice of the simulation box between $z-\Delta z / 2$ and $z+\Delta z / 2$ and $A$ is the cross section. The thickness $\Delta z$ was taken to be $\sigma / 5$.

The surface coverage of the flat plate by fluid molecules, $\Theta$, is defined as

$$
\begin{aligned}
\Theta & =\frac{(\text { Adsorption amount })}{\text { (Monolayer adsorption capacity) }} \\
& =\frac{A \int_{0}^{z_{0}} \rho(z) d z}{v_{\mathrm{m}}}=a_{\mathrm{m}} \int_{0}^{z_{0}} \rho(z) d z, \\
v_{\mathrm{m}} & =A / a_{\mathrm{m}}, \\
a_{\mathrm{m}} & =\frac{\sqrt{3}}{4}\left(4 / \rho_{\mathrm{L}}\right)^{2 / 3},
\end{aligned}
$$

where $z_{0}$ is the distance at which $\rho(z)$ exhibits the same density as the bulk vapor after several peaks, $v_{\mathrm{m}}$ is the molecular adsorption capacity, $a_{\mathrm{m}}$ is the molecular crosssectional area, and $\rho_{\mathrm{L}}$ is the density of the liquid in the bulk. Equation (12c) is based on the two assumptions: (i) the overall density of fluid molecules in the adsorbed film ranging from $z=0$ to $z=z_{0}$ is equal to that of the bulk liquid in equilibrium with the saturated vapor at given temperature, and (ii) the adsorbed molecules exhibit the fcc lattice configuration, the (111) plane of which coincides with the plane of the surface. The interplanar spacing of layers in the adsorbed film is equal to $\Delta_{\text {ads }}=\left(4 / \rho_{\mathrm{L}}\right)^{1 / 3} / \sqrt{3}$. The value of $\Theta \Delta_{\text {ads }}$ gives the effective thickness of the adsorbed film. 


\section{Surface forces}

As the sphere is located at $\mathbf{r}_{\mathrm{M}}=(0,0, D+R)$, the local density of fluid molecules is a function of $s=\left(x^{2}+y^{2}\right)^{1 / 2}$ as well as $z$,

$$
\rho^{*}(s, z)=\rho(s, z) \sigma^{3}, \quad \rho(s, z)=\langle N(s, z)\rangle / 2 \pi s \Delta s \Delta z,
$$

where $N(s, z)$ is the number of molecules in a cylindrical shell between $s-\Delta s / 2$ and $s+\Delta s / 2$ in the radial direction and between $z-\Delta z / 2$ and $z+\Delta z / 2$ in the central-axis direction. The thicknesses of $\Delta s=\Delta z=\sigma / 10$ were used.

The sphere-plate interaction force at distance $D$ is given by the sum of the forces acting on the sphere,

$$
\begin{aligned}
& \mathbf{F}(D)=\mathbf{F}^{\mathrm{solv}}(D)+\mathbf{F}^{\mathrm{dir}}(D), \\
& \mathbf{F}^{\mathrm{solv}}(D)=-\left\langle\sum_{i=1}^{N} \frac{d \phi_{\mathrm{MF}}\left(r_{\mathrm{M} i}\right)}{d r_{\mathrm{M} i}} \frac{\mathbf{r}_{\mathrm{M}}-\mathbf{r}_{i}}{\left|\mathbf{r}_{\mathrm{M}}-\mathbf{r}_{i}\right|}\right\rangle_{\mathbf{r}_{\mathrm{M}}=(0,0, D+R)},
\end{aligned}
$$

$$
\mathbf{F}^{\mathrm{dir}}(D)=-\frac{d U_{\mathrm{MW}}(D)}{d D} \boldsymbol{\delta}_{z}=\mathbf{0}, \quad \boldsymbol{\delta}_{z}=(0,0,1),
$$

where Eq. (14c) is derived from Eq. (5). The sphere-plate interaction force $\mathbf{F}(D)$ depends on the radius of the sphere $R$, but $\mathbf{F}(D) / 2 \pi R$ gives the force that is independent of $R .^{15}$ Hence, this normalized force is used in the followings unless otherwise specified.

The capillary excess of fluid molecules between the sphere and plate for the surface distance $D$ is defined as

$$
\Gamma(D)=\langle N(D)\rangle-\left\langle N\left(D_{\infty}\right)\right\rangle .
$$

Here $N(D)$ is the total number of fluid molecules in the box for the sphere-plate surface distance of $D$, and $D_{\infty}$ denotes the distance at which the adsorbed films on the sphere and the plate are regarded as isolated from each other. The value of $\Gamma(D)$ corresponds to the number of fluid molecules in a liquid bridge between the sphere and plate excluding the adsorbed films on the surfaces, as far as the effective adsorbed films do not overlap with each other (i.e., $D$ $\left.>2 \Theta \Delta_{\text {ads }}+\sigma\right)$.

\section{RESULTS AND DISCUSSION}

\section{A. Bulk vapors}

First of all, we aimed for the chemical potential $\mu$ that corresponds to the vapor-liquid coexistence at $T^{*}=1.0$. Kofke $^{29}$ reported the saturated pressure and the densities of the vapor and liquid phases at $T^{*}=1.0: p_{\text {sat }}^{*}=0.0242, \rho_{\mathrm{V}}^{*}$ $=0.0284$, and $\rho_{\mathrm{L}}^{*}=0.704$. Although Kofke did not report the corresponding chemical potential, Eq. (7) provides the approximated value, $\mu / k_{B} T=-11.53$ for $\mathrm{LJ}$ argon. We found that when $\mu / k_{B} T=-11.6585$ the results of our GCMC simulation agreed well with those abovementioned.

We computed $p$ and $\rho$ as a function of $\mu$ using the simulations. The results are shown in Fig. 2, where the results of the ideal gas assumption of Eqs. (7) and (8) are displayed for comparison. The results of the simulations are more asymptotic to those of the ideal gas assumption with decreasing $\mu / k_{B} T$; nonetheless, they show a discrepancy when

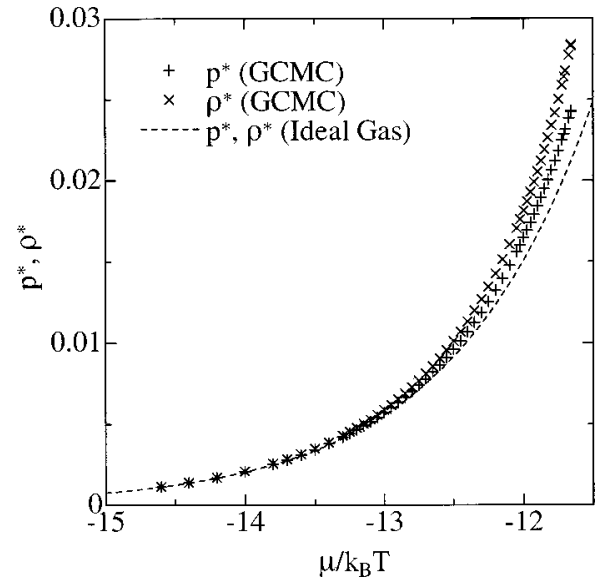

FIG. 2. Pressure and density of the bulk LJ fluid as a function of the chemical potential $\mu$ at $T^{*}=1.0$. The cross symbols are the GCMC results. The dashed line indicates the result by the ideal gas assumption of Eqs. (7) and (8): $p^{*}=\rho^{*}=\exp \left(\mu / k_{B} T\right) /(\Lambda / \sigma)^{3}$ for $T^{*}=1.0$.

$\mu / k_{B} T>-13$. This discrepancy becomes more striking when Fig. 2 is recasted as the $p-\rho$ relation in Fig. 3. On the other hand, the second-order virial equation, $p / \rho k_{B} T=1$ $+B_{2}(T) \rho$, gives a good description of the $p-\rho$ relation, where $B_{2}(T)$ is the second virial coefficient and equals $-5.3156 \sigma^{3}$ at $T^{*}=1.0 .^{30}$

Hereafter, to specify the simulation conditions we use the relative vapor pressure $p / p_{\text {sat }}$ and the bulk vapor density $\rho_{\text {bulk }}^{*}$, which are equivalent to $\mu$ as in Fig. 2 .

\section{B. Adsorption isotherms}

A typical profile of densities $\rho^{*}(z)$ is shown in Fig. 4, where the simulation conditions are the surface affinity of $\chi=1.0, p / p_{\text {sat }}=0.544$, and $\rho_{\text {bulk }}^{*}=0.0143$. This density profile indicates: (i) a significant number of fluid molecules are adsorbed on the interactive wall at $z / \sigma=0$; (ii) the density becomes equal to $\rho_{\text {bulk }}^{*}$ at $z / \sigma=7.1$ and then has a plateau; and (iii) the density near the hard wall at $z / \sigma=20$ is slightly smaller than $\rho_{\text {bulk }}^{*}$. We found that the density profiles $\rho^{*}(z)$

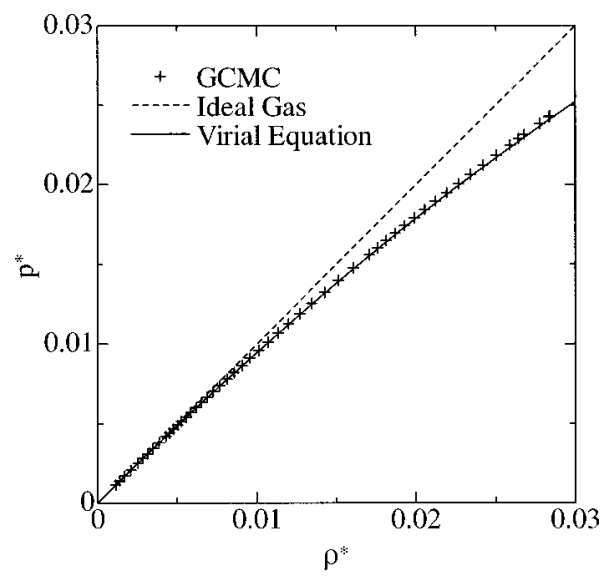

FIG. 3. Pressure-density relation of the bulk LJ fluid at $T^{*}=1.0$. The cross symbols are the GCMC results. The dashed line indicates the result by the ideal gas assumption of Eqs. (7) and (8): $p^{*}=\rho^{*}$ for $T^{*}=1.0$. The solid line shows the result by the second-order virial equation: $p^{*}=\rho^{*}$ (1 $-5.3156 \rho^{*}$ ) for $T^{*}=1.0$. For more details see the main text. 


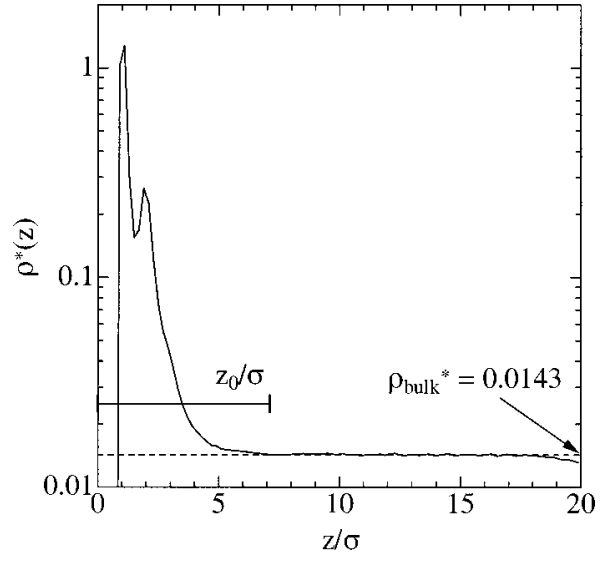

FIG. 4. Local density of fluid molecules as a function of the distance from the surface of $\chi=1.0$ (i.e., $\varepsilon_{\mathrm{WF}}=1.0 \varepsilon$ ) for $p / p_{\text {sat }}=0.544$ and $\rho_{\text {bulk }}^{*}$ $=0.0143$. The dashed line shows the density of the bulk fluid and $z_{0}$ indicates the distance at which $\rho^{*}(z)$ exhibits the same density as $\rho_{\text {bulk }}^{*}$ after several peaks.

had a similar plateau of $\rho_{\text {bulk }}^{*}$ even for simulations of the other conditions, indicating that the existence of the hard wall hardly affected the thickness of an adsorbed film (or $z_{0}$ ). Once $\rho^{*}(z)$ and $z_{0}$ are determined, the surface coverage $\Theta$ is given by Eq. (12); this parameter gives roughly the average number of adsorbed layers at the surface. In the case of Fig. 4 , we get $z_{0} / \sigma=7.1$ and $\Theta=1.14$.

The surface coverage $\Theta$ was calculated as a function of $p / p_{\text {sat }}$ for $\chi\left(=\varepsilon_{\mathrm{WF}} / \varepsilon\right)=0.5,0.7$, and 1.0 and the resulting adsorption isotherms are displayed in Fig. 5. The isotherms indicate that $\Theta$ increases with $p / p_{\text {sat }}$ and $\chi$. Further discussions of the isotherms are not the aim of the present paper but can be found elsewhere. ${ }^{31}$

\section{Surface forces}

A typical result of integrating averages of the sphereplate interaction force as a function of the $\mathrm{MC}$ cycle is depicted in Fig. 6, where the forces in all the directions become almost constant after 50000 cycles. At the final cycle, the forces in the $x$ and $y$ directions are equal to zero within

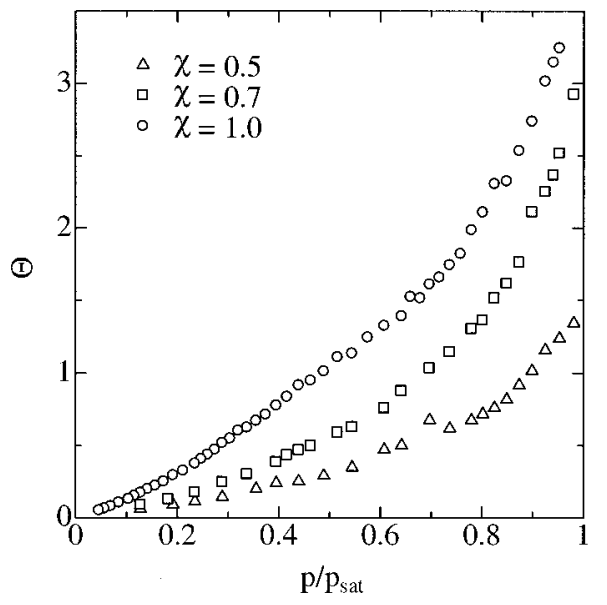

FIG. 5. Surface coverage as a function of the relative vapor pressure for $\chi=0.5,0.7$, and 1.0 (i.e., $\varepsilon_{\mathrm{WF}}=0.5 \varepsilon, 0.7 \varepsilon$, and $1.0 \varepsilon$ ).

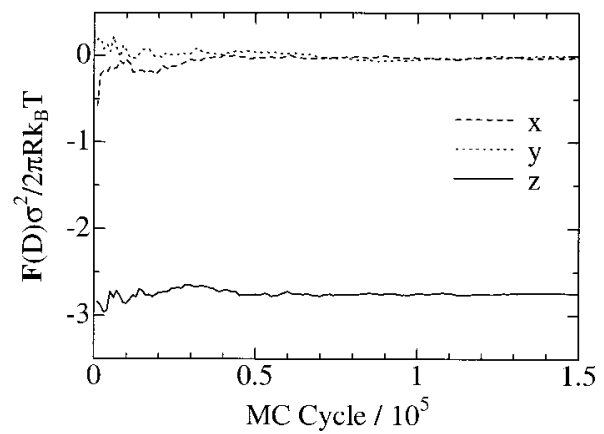

FIG. 6. Integrating averages of the sphere-plate interaction force as a function of the $\mathrm{MC}$ cycle after equilibration cycles. In this case, $\chi=1.0$ (i.e., $\left.\varepsilon_{\mathrm{MF}}=\varepsilon_{\mathrm{WF}}=1.0 \varepsilon\right), p / p_{\text {sat }}=0.544$, and $D / \sigma=2.0$. The dashed, dotted, and solid lines indicate the force in the $x, y$, and $z$ directions, respectively.

statistical accuracy, while the force in the $z$ direction exhibits a nonzero value because of the fluid-mediated interaction; similar results were obtained for simulations of the other conditions. It should be noted that the $x$ and $y$ components of the surface force may have nonzero values when the structured surfaces of the sphere and/or the plate are used. Hereafter, the $z$ component of $\mathbf{F}(D)$ is simply referred to as $F(D)$.

As mentioned above, the surface force $F(D)$ depends on the surface affinity $\chi$ and the relative vapor pressure $p / p_{\text {sat }}$ as well as the nearest separation of the surfaces $D$. In the following we investigate (1) the force as a function of $D$ and (2) the pull-off force as a function of $p / p_{\text {sat }}$.

\section{Force-distance profiles}

In the case of $\chi=1.0$, we evaluated the sphere-plate interaction force $F(D)$ and the capillary excess of fluid molecules $\Gamma(D)$ for $p / p_{\text {sat }}=0.234,0.544$, and 0.899 , which correspond to the surface coverage of $\Theta=0.37,1.14$, and 2.74, respectively as shown in Fig. 5. The results are displayed in Figs. 7 and 8. The forces in Figs. 7(a) and 7(b) become attractive at the distances less than $D / \sigma \approx 5.0$ for $p / p_{\text {sat }}$ $=0.234$ and $D / \sigma \approx 6.5$ for $p / p_{\text {sat }}=0.544$, respectively; otherwise, they remain zero. The capillary excesses in Figs. 8(a) and 8 (b) exhibit nonzero values at the distances less than $D / \sigma \approx 6.0$ for $p / p_{\text {sat }}=0.234$ and $D / \sigma \approx 8.0$ for $p / p_{\text {sat }}$ $=0.544$, respectively; otherwise, they are almost zero. These results indicate that the attractive force between the sphere and plate is caused by capillary condensation of vapor between the surfaces. For visualizing the capillary condensation, the local densities of fluid molecules near the sphere and plate are depicted in Fig. 9, where the darker color indicates a region of the higher density and the dotted line of a semicircle illustrates the origin of the sphere-fluid potential of Eq. (4), while the origin of the plate-fluid potential of Eq. (2) is the line of $z=0$ (i.e., the $s$ axis). In rightward three panels of Figs. 9(a) and 9(b) the capillary condensation is invisible; however, it does more or less take place in several of the panels as expected from the results of Figs. 8(a) and 8(b). On the other hand, leftward three panels of Figs. 9(a) and 9 (b) reveal that the capillary condensation becomes 

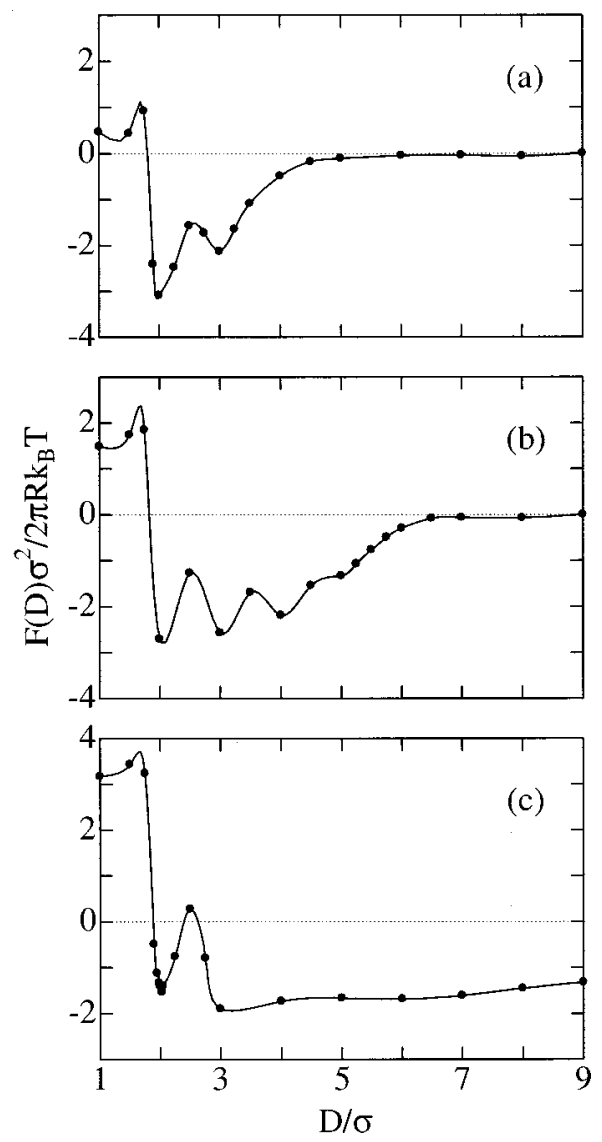

FIG. 7. Force between the sphere and plate as a function of the nearest separation of the surfaces for (a) $p / p_{\text {sat }}=0.234$, (b) $p / p_{\text {sat }}=0.544$, and (c) $p / p_{\text {sat }}=0.899$. In all cases, $\chi=1.0$ (i.e., $\varepsilon_{\mathrm{MF}}=\varepsilon_{\mathrm{WF}}=1.0 \varepsilon$ ). The solid lines are the guides for the eyes.

more significant to form a larger liquid bridge in the gap between the sphere and plate as the surface distance is reduced less than the capillary transition point $D_{\mathrm{t}}$, where $D_{\mathrm{t}} / \sigma \approx 4.0$ for $p / p_{\text {sat }}=0.234$ and $D_{\mathrm{t}} / \sigma \approx 6.0$ for $p / p_{\text {sat }}$ $=0.544$. This behavior is consistent with that in Figs. 8(a) and $8(\mathrm{~b})$, where the capillary excess remarkably increases with decreasing the surface distance less than $D \approx D_{\mathrm{t}}$. Thus, when the sphere and the plate come closer to each other, first capillary condensation of a vapor takes place in the gap between the surfaces; second the force between the sphere and plate becomes attractive; and third the capillary condensed fluid experiences vapor-to-liquid transition to form a liquid bridge between the surfaces. These results are in fair agreement with those of force measurements ${ }^{18,19,32-34}$ and MD simulations. ${ }^{21,22}$ No transition point was found for $p / p_{\text {sat }}$ $=0.899$ in the range of $D / \sigma \leqslant 9.0$, where a liquid bridge was formed. Comparison of these results for different vapor pressures in Figs. 7-9 indicates that as the relative vapor pressure increases, the onset of the attractive force due to capillary condensation between the surfaces becomes farther and the resultant force has a gentler slope. This agrees well with the results from the SFA (Ref. 33) and the DFT. ${ }^{16}$

Another noteworthy point in Fig. 7 is that in the short range of $D / \sigma<4.0$ the force significantly oscillates with periodicity of about $\sigma$ (=the diameter of a fluid molecule); this
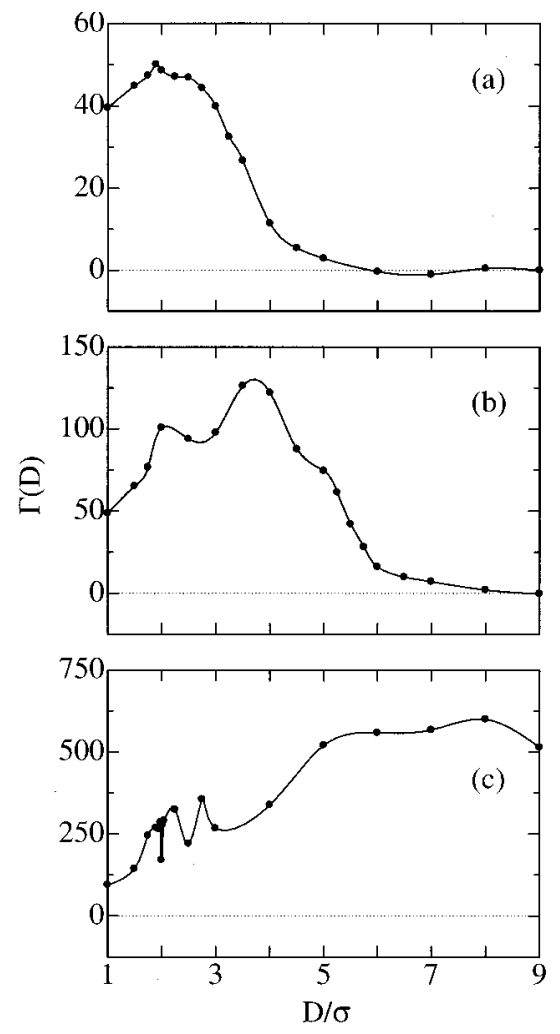

FIG. 8. Capillary excess of fluid molecules in the gap between the sphere and plate as a function of the nearest separation of the surfaces for (a) $p / p_{\text {sat }}=0.234$, (b) $p / p_{\text {sat }}=0.544$, and (c) $p / p_{\text {sat }}=0.899$. In all cases, $\chi$ $=1.0$ (i.e., $\varepsilon_{\mathrm{MF}}=\varepsilon_{\mathrm{WF}}=1.0 \varepsilon$ ). The solid lines are the guides for the eyes.

oscillation is attributed to the packing effect of the fluid molecules condensed between the surfaces. Similar behavior was observed in SFA measurements, ${ }^{18,19}$ DFT analyses, ${ }^{16}$ and MD simulations. ${ }^{22}$ The force in Fig. 7 exhibits a global minimum at $D / \sigma \approx 2$ for $p / p_{\text {sat }}=0.234$ and 0.544 and at $D / \sigma$ $\approx 3$ for $p / p_{\text {sat }}=0.899$; this maximum attraction corresponds to the pull-off force. Note that there are zero layers of solvent between the surfaces at $D / \sigma \approx 1$, one layer at $D / \sigma \approx 2$, two layers at $D / \sigma \approx 3$, and so forth. The pull-off distances of $D / \sigma \approx 2$ and 3 indicate that the sphere and the plate prefer being separated by at least one layer of solvent to being in contact with each other under the conditions of our simulations where the direct sphere-plate interaction is negligible compared to fluid-fluid, sphere-fluid, and plate-fluid interactions.

As explained in Sec. III C, interpretation of the capillary excess in Fig. 8 is complicated by overlap and compression of the effective adsorbed layers on the surfaces when $D / \sigma$ $\leqslant\left(2 \Theta \Delta_{\text {ads }}+\sigma\right) / \sigma=1.8, \quad 3.4$, and 6.7 for $p / p_{\text {sat }}=0.234$, 0.544 , and 0.899 , respectively. Otherwise, the capillary excess, which increases with decreasing $D$ as in Fig. 8, gives the approximate number of the fluid molecules in a liquid bridge between the sphere and plate. This suggests that the volume of the liquid bridge varies with the distance between the surfaces; however, the isovolume assumption has been made in most of the capillary force models. ${ }^{5,15,35-37}$ 


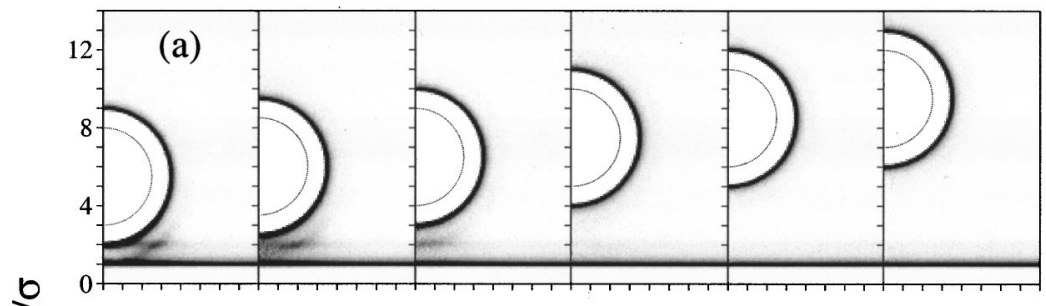

$\frac{b}{N}$

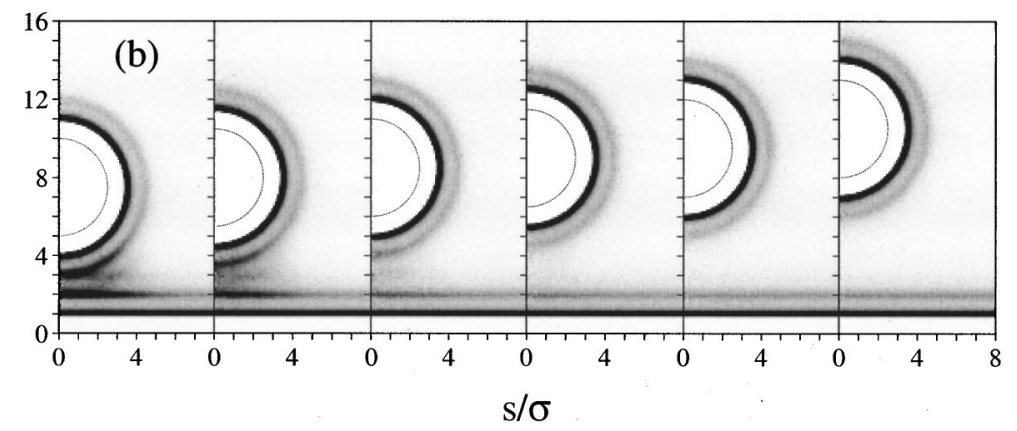

FIG. 9. Contour maps of the fluid density near the sphere and plate for different separations. The top row of panels (a), $p / p_{\text {sat }}=0.234$; and the bottom row (b), $p / p_{\text {sat }}=0.544$. The panels in each row from left to right correspond to the separations: $D / \sigma=3.0,3.5,4.0,5.0$, 6.0, and 7.0 for (a); and $D / \sigma=5.0,5.5,6.0,6.5,7.0$, and 8.0 for (b). In all cases, $\chi=1.0$ (i.e., $\varepsilon_{\mathrm{MF}}=\varepsilon_{\mathrm{WF}}$ $=1.0 \varepsilon$ ). The darker color indicates a region of the higher density. The dotted lines of a semicircle illustrate the origin of the sphere-fluid potential of Eq. (4), while the origin of the plate-fluid potential of Eq. (2) is the solid lines of $z=0$ (i.e., the $s$ axis). For more details see the main text.

\section{Pull-off forces}

As shown in Fig. 7, the pull-off forces were observed at $D / \sigma \approx 2$ or 3 in our simulations; the pull-off distance and the magnitude of the pull-off force will largely depend on the surface affinity $\chi$ as well as $p / p_{\text {sat }}$. The pull-off force as a function of $p / p_{\text {sat }}$ was calculated for $\chi=0.5,0.7$, and 1.0 (i.e., $\varepsilon_{\mathrm{MF}}=\varepsilon_{\mathrm{WF}}=0.5 \varepsilon, 0.7 \varepsilon$, and $1.0 \varepsilon$ ). The results are shown in Fig. 10, where the dashed line indicates the contribution of the Laplace pressure to the pull-off force, that is $F / 2 \pi R=-2 \gamma_{\mathrm{LV}} \cos \theta ;{ }^{15} \theta$ is the contact angle of a liquid on a surface (i.e., $\theta=0^{\circ}$ is assumed) and $\gamma_{\mathrm{LV}}$ is the liquidvapor interfacial tension (i.e., $\gamma_{\mathrm{LV}}^{*}=\gamma_{\mathrm{LV}} \sigma^{2} / \varepsilon=0.450$ for the LJ fluid at $\left.T^{*}=1.0\right) .{ }^{38}$ Figure 10 demonstrates that the pulloff force profiles exhibit a peak under the conditions of our simulations: the peak force is larger than the Laplace force. This is in good agreement with the results of the DFT analysis for a similar system ${ }^{16}$ and of AFM measurements in a

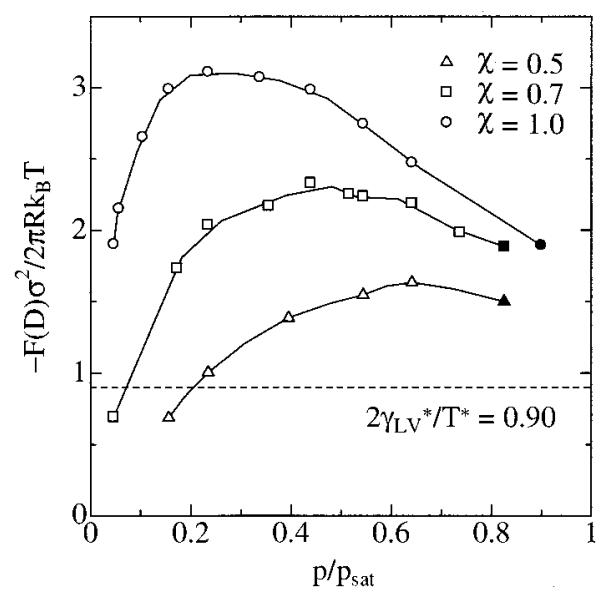

FIG. 10. Pull-off force between the sphere and plate as a function of the relative vapor pressure for $\chi=0.5,0.7$, and 1.0 (i.e., $\varepsilon_{\mathrm{MF}}=\varepsilon_{\mathrm{WF}}$ $=0.5 \varepsilon, 0.7 \varepsilon$, and $1.0 \varepsilon$ ). The open and filled symbols in the plots indicate the different pull-off distances: open symbols, one layer of solvent at $D / \sigma$ $\approx 2$; and filled symbols, two layers of solvent at $D / \sigma \approx 3$. The solid lines are the guides for the eyes. The dashed line indicates the Laplace force. For more details see the main text. humid environment, ${ }^{4,5}$ except for the constant pull-off force in the range of the low vapor pressures where the direct sphere-plate interaction becomes important but is not considered in our GCMC simulations. It is interesting to note that analogous behavior was found in a binary mixture; the pull-off forces in alcohol-water mixtures had a peak at an alcohol-rich concentration according to AFM (Refs. 39 and 40) and MD studies. ${ }^{24}$ While other studies ${ }^{7,16,19}$ have shown that the pull-off force tends to the Laplace force of $F / 2 \pi R$ $=-2 \gamma_{\mathrm{LV}} \cos \theta$ as $p / p_{\text {sat }} \rightarrow 1$, it is unclear whether this limiting value is obtained in Fig. 10 or not. This could be an effect of the rather small sphere used in the present study; however, the detailed examination is our future study. As both $\varepsilon_{\mathrm{MF}}$ and $\varepsilon_{\mathrm{WF}}$ decrease, the position of the peak in Fig. 10 shifts to a higher relative pressure and the height of the peak becomes smaller. Judging from the shifts of the peak position, the still smaller values of $\varepsilon_{\mathrm{MF}}$ and $\varepsilon_{\mathrm{WF}}$ would result in disappear of the peak, that is, a monotonic increase of the pull-off force with the relative vapor pressure. In fact this type of the pull-off force profile was obtained by the DFT for weak surface-fluid interactions. ${ }^{16}$

The pull-off forces are plotted in Fig. 11 as a function of

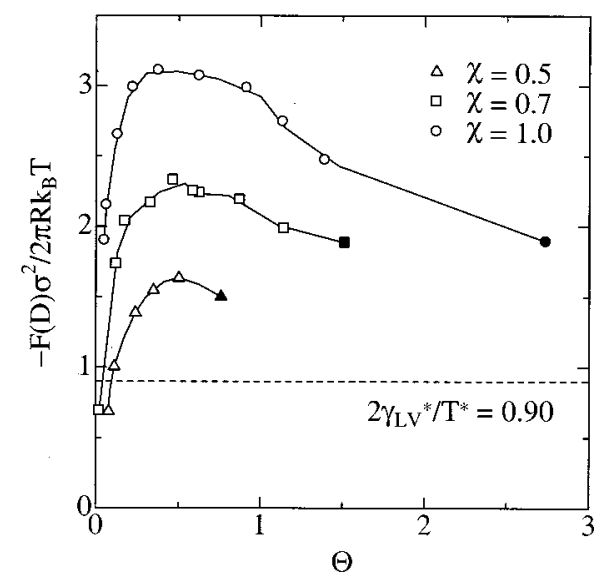

FIG. 11. Same as Fig. 10 but plotted as a function of the surface coverage. 

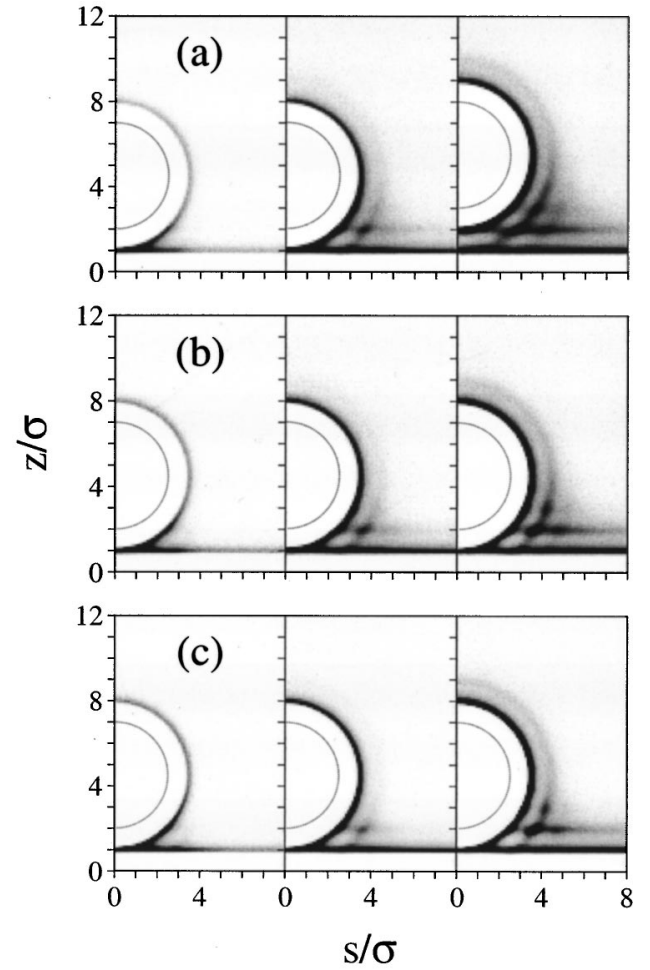

FIG. 12. Contour maps of the fluid density near the sphere and plate at the pull-off distance for different relative vapor pressures. The top row of panels (a), $\chi=0.5$; the middle row (b), $\chi=0.7$; and the bottom row (c), $\chi=1.0$. The panels in the row from left to right correspond to the relative pressures (or the surface coverages): $p / p_{\text {sat }}=0.234,0.641$, and 0.825 (or $\Theta=0.11$, 0.50 , and 0.76 ) for the top row (a); $p / p_{\text {sat }}=0.173,0.439$, and 0.641 (or $\Theta$ $=0.12,0.47$, and 0.88$)$ for the middle row (b); and $p / p_{\text {sat }}=0.104,0.234$, and 0.439 (or $\Theta=0.13,0.37$, and 0.91 ) for the bottom row (c). The central panel in each row corresponds to the peak of the pull-off force in Fig. 10 (or Fig. 11). For more details see the caption of Fig. 9.

the surface coverage $\Theta$ instead of the relative vapor pressure $p / p_{\text {sat }}$, where $\Theta$ was obtained for given $p / p_{\text {sat }}$ with the help of Fig. 5. It should be noted that the coverage of the planar surface does not coincide, in the strict sense, with that of the curved surface. Figure 11 reveals that the pull-off force has a peak at $\Theta \approx 0.45$ regardless of the surface affinity $\chi$. This scaling feature by the surface coverage in a vapor is similar to that by the relative water content in alcoholic liquids. ${ }^{40}$ To elucidate the wetting features of the sphere and plate at the pull-off distance (i.e., $D / \sigma \approx 2$ or 3 ), Fig. 12 displays the local densities of fluid molecules for different relative pressures (or surface coverages). The peaks of the pull-off force in Fig. 10 (or Fig. 11) correspond to the central panels of Figs. 12(a)-12(c); these three panels for $\Theta \approx 0.45$ exhibit the same feature of a liquid bridge, that is, a circular string of fluid molecules plus an adsorbed layer on each surface. In leftward panels of Figs. 12(a)-12(c) for the smaller values of $\Theta$, a significant number of fluid molecules are observed near the gap between the sphere and plate, compared with the surfaces far from the contact area; however, no effective bridge is formed near the gap. On the other hand, in rightward panels of Figs. 12(a)-12(c) for the larger values of $\Theta$, the liquid bridge becomes enlarged. Thus, a circular string of fluid molecules is found to be necessary and sufficient for a liquid bridge between the surfaces at the pull-off distance.

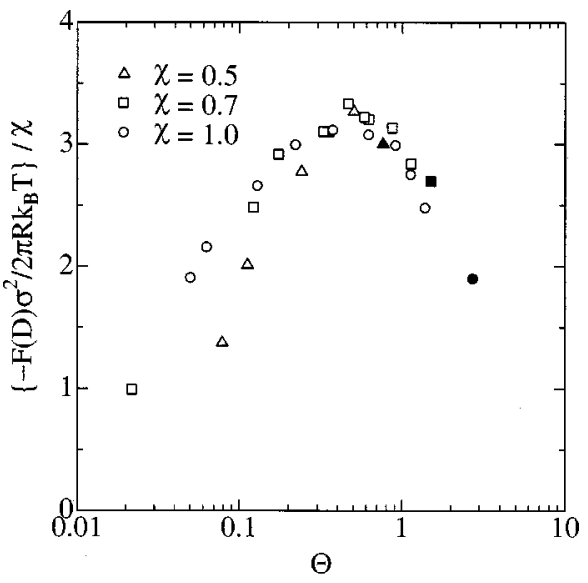

FIG. 13. Same as Fig. 11 but the pull-off forces are normalized by the surface affinity $\chi$ and plotted on a log scale of the surface coverage.

As described above, a molecular-string meniscus plus adsorbed layers appears to give a peak of the pull-off force when $\Theta \approx 0.45$; otherwise, the meniscus is unsaturated or supersaturated, which leads to the reduction of the pull-off force. For this reason, if the surface-fluid interactions are weakly attractive enough to keep the surface coverage $\Theta$ less than $\approx 0.45$ up to the high relative vapor pressure $p / p_{\text {sat }}$ (but ideally $\Theta \rightarrow \infty$ for $p / p_{\text {sat }} \rightarrow 1$ ), the pull-off force seems to increase monotonically with $p / p_{\text {sat }}$ as aforementioned because of the unsaturated meniscus. In contrast, if the surface-fluid interactions are strongly attractive, it is expected that the meniscus becomes saturated at the extremely low relative pressure (i.e., $p / p_{\text {sat }} \approx 0$ ) and consequently the pull-off force shows an apparently monotonic decrease with the relative pressure. It should be noted again that in the present study the pull-off force includes no effects of the direct sphere-plate interaction. Addition of the direct sphere-plate interaction alters both the pull-off distance and the resultant force as reported in the DFT study. ${ }^{16}$ Especially when the direct interaction between the surfaces of the sphere and plate is even more attractive than fluid-fluid and surface-fluid interactions, it dominates the pull-off force in which the fluid-mediated force is negligible [see Eq. (14a)]. In this case, the pull-off force would be observed at the distance where the direct sphere-plate interaction becomes most attractive and no fluid layers are interposed between the surfaces; this pull-off force would result in a constant value regardless of the relative pressure. Unfortunately, it takes a great deal of computation time to investigate the effects of the direct sphere-plate interaction on the pull-off force by GCMC simulations other than the DFT; ${ }^{16}$ therefore, this investigation is our future study.

Finally, the pull-off forces in Fig. 11 were normalized by the surface affinity $\chi$ and plotted on a log scale of the surface coverage as shown in Fig. 13. Interestingly, all the data seem to be on a master curve; this result coincides with the similarity of the wetting features among Figs. 12(a)-12(c). This leads to an important suggestion: as far as the effect of the direct sphere-plate interaction on the pull-off force is trivial, the wetting feature at the pull-off distance and the resultant 
pull-off force can be entirely explained by the surface coverage and the surface affinity.

\section{CONCLUSIONS}

In this article, we have investigated the interaction forces between a rigid nanosphere and a flat plate in a capillarycondensable vapor using GCMC simulations. The following conclusions are drawn:

(1) The force between the sphere and plate becomes attractive because of capillary condensation between the surfaces. The onset of the attractive force becomes farther as the relative vapor pressure increases. After capillary condensation, a liquid bridge forms in the gap between the sphere and plate and its volume varies with the distance between the surfaces.

(2) In the short range, the force-distance profile shows an oscillation with periodicity of about the diameter of a fluid molecule and exhibits a global minimum, the position and the magnitude of which correspond to the pull-off distance and the pull-off force, respectively.

(3) The curve of the pull-off force as a function of the relative vapor pressure has a peak. The peak position shifts to a higher relative pressure and the peak height becomes smaller, with decreasing the attractive interaction of the surfaces of the sphere and the plate with a fluid molecule.

(4) At the relative vapor pressure where the pull-off force becomes maximum, the coverage of the surface by fluid molecules is found to be $\approx 0.45$ regardless of the strength of the surface-fluid attraction; in addition, the gap between the sphere and plate exhibits the same wetting feature, that is, a circular string of fluid molecules plus an adsorbed layer on each surface.

(5) Fluid contribution to the pull-off force could be described by a master curve, that is, the force scaled by the strength of the surface-fluid attraction and the coverage of the surface by fluid molecules.

Our force-distance profiles almost coincide with those from the DFT analyses for similar systems; ${ }^{16,17}$ also, the force profiles are in fair agreement with those from SFA (Refs. 18, 19, 32, 33) and AFM (Ref. 34) measurements and MD simulations. ${ }^{21,22}$ Similar curve of the pull-off force as a function of the relative vapor pressure was observed in the DFT analysis ${ }^{16}$ and in AFM measurements for tip-substrate interactions in a humid environment. ${ }^{4,5}$ The surface coverage defined by Eq. (12) is not very clean as a scaling parameter for the corresponding relative vapor pressure, because it gives only a rough estimate for the number of adsorbed layers at the surface. However, this parameter turned out to be useful for scaling the results as demonstrated in Figs. 10 and 11 .
${ }^{1}$ G. Binning, C. F. Quate, and C. Gerber, Phys. Rev. Lett. 56, 930 (1986).

${ }^{2}$ W. A. Ducker, T. J. Senden, and R. M. Pashley, Nature (London) 353, 239 (1991); Langmuir 8, 1831 (1992).

${ }^{3}$ J. N. Israelachvili and G. E. Adams, J. Chem. Soc., Faraday Trans. 1 74, 975 (1978).

${ }^{4}$ L. Xu, A. Lio, J. Hu, D. F. Ogletree, and M. Salmeron, J. Phys. Chem. B 102, 540 (1998)

${ }^{5}$ M. He, A. S. Blum, D. E. Aston, C. Buenviaje, R. M. Overney, and R. Luginbuhl, J. Chem. Phys. 114, 1355 (2001).

${ }^{6}$ M. Fuji, K. Machida, T. Takei, T. Watanabe, and M. Chikazawa, J. Phys. Chem. B 102, 8782 (1998).

${ }^{7}$ H. K. Christenson, J. Colloid Interface Sci. 121, 170 (1988).

${ }^{8}$ L. R. Fisher and J. N. Israelachvili, Colloids Surf. 3, 303 (1981).

${ }^{9}$ H. K. Christenson, Langmuir 12, 1404 (1996).

${ }^{10}$ H. K. Christenson, J. Phys. Chem. 97, 12034 (1993).

${ }^{11}$ M. M. Kohonen and H. K. Christenson, Langmuir 16, 7285 (2000).

${ }^{12}$ K. L. Johnson, K. Kendall, and A. D. Roberts, Proc. R. Soc. London, Ser. A 324, 301 (1971).

${ }^{13}$ A. Fogden and L. R. White, J. Colloid Interface Sci. 138, 414 (1990).

${ }^{14}$ D. Maugis, J. Chem. Phys. 150, 243 (1992).

${ }^{15}$ J. N. Israelachvili, Intermolecular and Surface Forces, 2nd ed. (Academic, New York, 1991).

${ }^{16}$ L. J. D. Frink and F. van Swol, J. Chem. Phys. 106, 3782 (1997).

${ }^{17}$ C. Bauer, T. Bieker, and S. Dietrich, Phys. Rev. E 62, 5324 (2000).

${ }^{18}$ E. J. Wanless and H. K. Christenson, J. Chem. Phys. 101, 4260 (1994).

${ }^{19}$ H. K. Christenson and V. V. Yaminsky, Langmuir 9, 2448 (1993).

${ }^{20}$ M. P. Allen and D. J. Tildesley, Computer Simulation of Liquids (Clarendon, Oxford, 1987).

${ }^{21}$ E. J. W. Wensink, A. C. Hoffmann, M. E. F. Apol, and H. J. C. Berendsen, Langmuir 16, 7392 (2000).

${ }^{22}$ W. D. Luedtke and U. Landman, Comput. Mater. Sci. 1, 1 (1992); U. Landman, W. D. Luedtke, J. Ouyang, and T. K. Xia, Jpn. J. Appl. Phys., Part 1 32, 1444 (1993).

${ }^{23}$ H. Shinto, M. Miyahara, and K. Higashitani, J. Colloid Interface Sci. 209, 79 (1999).

${ }^{24}$ H. Shinto, M. Miyahara, and K. Higashitani, Langmuir 16, 3361 (2000).

${ }^{25}$ L. D. Gelb and R. M. Lynden-Bell, Phys. Rev. B 49, 2058 (1994).

${ }^{26}$ W. A. Steele, Surf. Sci. 36, 317 (1973).

${ }^{27}$ H. C. Hamaker, Physica (Amsterdam) 4, 1058 (1937).

${ }^{28}$ D. J. Adams, Mol. Phys. 29, 307 (1975); 32, 647 (1976).

${ }^{29}$ D. A. Kofke, J. Chem. Phys. 98, 4149 (1993).

${ }^{30}$ J. J. Nicolas, K. E. Gubbins, W. B. Street, and D. J. Tildesley, Mol. Phys. 37, 1429 (1979).

${ }^{31}$ L. D. Gelb, K. E. Gubbins, R. Radhakrishnan, and M. SliwinskaBartkowiak, Rep. Prog. Phys. 62, 1573 (1999), and references therein; 63, 727(E) (2000)

${ }^{32}$ J. Crassous, E. Charlaix, H. Gayvallet, and J.-L. Loubet, Langmuir 9, 1995 (1993).

${ }^{33}$ J. Crassous, E. Charlaix, and J.-L. Loubet, Europhys. Lett. 28, 37 (1994).

${ }^{34}$ M. Binggeli and C. M. Mate, Appl. Phys. Lett. 65, 415 (1994).

${ }^{35}$ W. C. Clark, J. M. Haynes, and G. Mason, Chem. Eng. Sci. 23, 810 (1968)

${ }^{36}$ M. A. Erle, D. C. Dyson, and N. R. Morrow, AIChE J. 17, 115 (1971).

${ }^{37}$ F. M. Orr, L. E. Scriven, and A. P. Rivas, J. Fluid Mech. 67, 723 (1975).

${ }^{38}$ A. Trokhymchuk and J. Alejandre, J. Chem. Phys. 111, 8510 (1999).

${ }^{39}$ Y. Kanda, T. Nakamura, and K. Higashitani, Colloids Surf., A 139, 55 (1998).

${ }^{40}$ Y. Kanda, S. Iwasaki, and K. Higashitani, J. Colloid Interface Sci. 216, 394 (1999). 